\title{
Impact of Mycobacterium tuberculosis complex lineages as a determinant of disease phenotypes from an immigrant rich moderate tuberculosis burden country
}

Bright Varghese ${ }^{1}$, Mushira Enani ${ }^{2}$, Abdulrahman Alrajhi ${ }^{3}$, Sameera Al Johani ${ }^{4}$, Ali Albarak ${ }^{5}$, Sahar Althawadi ${ }^{6}$, Noura Elkhizzi ${ }^{7}$, Hawra AlGhafli ${ }^{1}$, Mohammed Shoukri ${ }^{8}$ and Sahal Al - Hajoj ${ }^{1^{*}}$

\begin{abstract}
Background: Growing evidences suggested that the Mycobacterium tuberculosis complex (MTBC) lineages can determine the clinical outcome of pulmonary and extra-pulmonary tuberculosis. However, limited data only available revealing such association of bacterial genotypes and clinical phenotypes from immigrant rich countries.

Methods: A multicenter study has been carried out on a collection of 2092 (1003 extrapulmonary and 1089 pulmonary) MTBC isolates. Genotyping of all the isolates were carried out by spoligotyping and 24 loci based MIRU-VNTR typing.

Results: Demographically domination of young Saudi nationals (61.4\%) and men (61.2\%) were found in this cohort. Lymph nodes (62.4\%) and gastrointestinal sites (16.7\%) were the most common anatomical sites of infection. The predominant lineages were Delhi/CAS (26.9\%), EAI (14.2\%) and Ghana (9.9\%). Mycobacterium africanum type I and II were reported for the first time in the country among extrapulmonary cases. 'Ancestral' lineages M.bovis (OR-5.22; 95\% Cl-2.23-8.22, $p-<0.001)$ and Delhi/CAS (OR-0.57; 95\% Cl-0.411-0.734, $p-<0.001)$ were directly associated with lymph node tuberculosis and gastrointestinal tuberculosis (M. bovis-OR-0.33; 95\% Cl-0.085-0.567, p-0.001 and Delhi/CAS-OR-1.87; 95\% Cl-1.22-2.53, p-<0.001) respectively. Among the 'Modern' lineages, EAl showed significant association to central nervous system tuberculosis (OR-1.98; 95\% Cl-0.76-3.19, p-0.04) and Uganda-I to gastrointestinal tuberculosis (OR-2.41; 95\% Cl-0.77-4.06, p-0.02).
\end{abstract}

Conclusions: The findings substantially contribute to the emerging evidences that MTBC lineages influence disease phenotypes and epidemiological consequences.

Keywords: Mycobacterium tuberculosis complex, Saudi Arabia, Disease phenotypes, Tuberculosis, Extrapulmonary tuberculosis

\section{Background}

Tuberculosis (TB) caused by Mycobacterium tuberculosis complex (MTBC) remains as a major public health challenge despite of implementing several control programs [1]. World Health Organization (WHO) estimated in 2016 that, there were 10.6 million newly diagnosed TB cases including 490,000 multidrug resistant cases and 1.4 million deaths [1]. The MTBC primarily infects the

\footnotetext{
* Correspondence: hajoj@kfshrc.edu.sa

'Department of Infection and Immunity, MBC-03, King Faisal Specialist Hospital and Research Centre, Post Box \# 3354, Riyadh 11211, Saudi Arabia Full list of author information is available at the end of the article
}

lungs, but virtually can affect any site in the body. Extrapulmonary $\mathrm{TB}$ (EPTB) is a difficult form of $\mathrm{TB}$ to diagnose and treat and results in high mortality and morbidity. Recent national level report from Saudi Arabia, showed $25.6 \%$ of annually reported new TB cases were EPTB [2]. Interestingly, the annual EPTB incidence rate in Saudi Arabia is comparatively higher than developed countries, where ЕРTB incidences are even increasing steadily $[3,4]$.

In EPTB, lymph nodes, meninges, kidney, spine and joints/bones are mostly affected. However, TB in pericardium, peritoneum, pleura, liver, intestine, skin, genitourinary

(c) The Author(s). 2018 Open Access This article is distributed under the terms of the Creative Commons Attribution 4.0 International License (http://creativecommons.org/licenses/by/4.0/), which permits unrestricted use, distribution, and 
tracts, spleen, ileum, cecum, eyes, breast, penis, are also occasionally reported [5]. EPTB is mostly observed as a key indicator of immunosuppression. To date, bacterial determinants of pulmonary TB (PTB) or EPTB have not been clearly estimated, although large attention is directed to explore the host and pathogen determinants. There have been controversies in correlating specific lineages with morbidity and mortality due to TB infection. Several previous studies revealed conflicting evidences on the association of MTBC phylogenetic lineages and site of infection [6, 7]. However, such analysis was scarce from the Middle Eastern countries including Saudi Arabia. Saudi Arabia has a highly diverse population structure including 10.4 million immigrant workers from around the world. In addition, annually the country receives 10 million pilgrims to the Islamic holy cities. This population diversity was reflected highly on the spectrum of MTBC lineages in the country, with the presence of almost all defined lineages including indigenous strains [8-10]. Although, a recent study reported the lineage spectrum of MTBC in PTB and EPTB cases in the country, no detailed systematic analysis was carried out to date [8]. Therefore, for the first time in the country a multicenter study on a large cohort of pulmonary and extrapulmonary TB cases has been carried out to analyze the impact of MTBC lineages towards disease phenotypes.

\section{Methods}

\section{Study design}

The study has been carried out in four major referral hospitals in Riyadh (Central Province). During August 2014-July 2016, all cases diagnosed with active TB and culture positivity were included. A collection of 1003 non-repetitive extrapulmonary MTBC (M.tuberculosis, M.bovis, M.africanum) culture isolates were successfully enrolled. In addition, 1089 pulmonary MTBC isolates from sputum, gastric aspirates, bronchioalveolar lavage and lung tissues were included as a control. Standard data collection form with information on age, gender, nationality, infection site, AFB smear results and HIV status were filled during the isolate collection from each study centers. All culture negative cases were excluded from enrollment. Disseminated TB and miliary TB cases were also excluded, while pleural TB cases were included as PTB. The study has been reviewed and approved by the Office of Research Affairs at King Faisal Specialist Hospital and Research Centre, Riyadh.

\section{Sample collection, processing, and identification}

Mycobacterium tuberculosis complex isolates from extrapulmonary sites were collected from EPTB diagnosed cases in the participating four study sites. Pulmonary isolates were selected from the culture collection of the research facility. Genomic DNA was extracted from all the isolates by using PrepIT MAX kit (DNA Genotek,
Ottawa, Canada) according to the manufacturer's instructions. Primary genotyping of all isolates were carried out by using the commercially available spoligotyping assay (Ocimum Biosolutions, Hyderabad, India). The 24 loci based MIRU-VNTR typing based on quadruplex PCR (Genoscreen, Lille, France) was carried out according to the manufacturer's instructions in a 3730xl DNA Analyzer (Life Technologies, CA, USA).

\section{Data analysis \\ Definition of clinical phenotypes}

Two major grouping of isolates were followed based on the site of infection, pulmonary and extrapulmonary. Based on the proportion of cases, extrapulmonary group were further classified into six groups namely lymph nodes, gastrointestinal (gastrointestinal tract, solid visceral organs), central nervous systems (CNS), bone and joints, skin, and urogenital. All other sites with lower proportions were grouped into "others".

\section{Genotyping data}

The spoligotyping membranes were scanned and data were converted into numerical octal codes. The alleles of MIRU-VNTR types were primarily identified by using the Genemapper version-4.0 (Applied Biosystems, CA, USA). The spoligo-octal signatures and the MIRUVNTR allele profiles were combined in Microsoft Excel and submitted to the international online MIRU_VNTR database (www.miru-vntrplus.org) for a combined bestmatch and phylogenetic tree-based analysis. We followed the phylogenetic classification based on large sequence polymorphisms to make combined discussion of study findings. The 'Ancestral' lineages included Delhi/CAS, M. bovis and M. africanum, while 'Modern' lineages included EAI, Beijing and Euro-American respectively [11].

The statistical analysis of data was carried out by using the SPSS version-20 package (IBM, NY, USA). Major strain lineages and different sites of infections were compared by Chi square test and regression analysis, estimating the odds ratio and 95\% confidence interval. The $p$ value $\leq 0.05$ was considered as statistically significant.

\section{Results}

During the study period, 1003 extrapulmonary isolates and 1089 pulmonary isolates were enrolled. Matching the age group of patients against the PTB and EPTB incidence showed significant variations with statistical associations. The age group below 14 years showed a statistically significant predominance of EPTB $(P$ value $<0.0001)$. On the other hand, PTB incidence was more significant among the age group 15-29 and 49-59. Overall, $76.6 \%$ of EPTB cases were found with an age below 45 years. Among, EPTB patients $83.3 \%$ were Saudi 
nationals. Demographics and other patient characteristics have been summarized in Table 1 and Additional file 1 . Distribution major lineages among the study population based on their nationality is depicted in Additional file 2.

\section{Site of extrapulmonary TB infections}

The reported 1003 EPTB cases were defined into six major groups with a predominance of lymph nodes (62.4\%). Other major sites were, gastrointestinal (16.7\%), central nervous system (8.6\%), genitourinary system (4.1\%), skin and soft tissues (2.2\%), bone and joints $(4.5 \%)$ and remaining $1.5 \%$ were other rare sites. The cervical (43.6\%), axillary (35.9\%) and supraclavicular (17.2\%) lymph nodes were generally infected. The diversity of infection sites among the study cohort was depicted in Fig. 1.

\section{Phylogenetic diversity of MTBC isolates of extrapulmonary sites}

The MTBC isolates causing EPTB showed a highly diverse lineage spectrum with the presence of all 5 major

Table 1 Demographic and clinical summary of study subjects

\begin{tabular}{|c|c|c|c|}
\hline \multirow[t]{2}{*}{ Parameters } & \multirow{2}{*}{$\begin{array}{l}\text { Extrapulmonary } \\
N(\%) \\
N=1003\end{array}$} & \multirow{2}{*}{$\begin{array}{l}\text { Pulmonary } \\
N(\%) \\
N=1089\end{array}$} & \multirow[t]{2}{*}{$P$ value } \\
\hline & & & \\
\hline \multicolumn{4}{|l|}{ Age group } \\
\hline $0-14$ & $130(12.9)$ & $28(2.6)$ & $<0.0001$ \\
\hline $15-29$ & $321(32.1)$ & 418 (38.4) & 0.002 \\
\hline $30-45$ & 317 (31.6) & $323(29.7)$ & 0.33 \\
\hline $49-59$ & $136(13.5)$ & $215(19.7)$ & 0.002 \\
\hline$>60$ & $99(9.9)$ & $105(9.6)$ & 0.86 \\
\hline Nationality & & & $<0.0001$ \\
\hline Saudi & 835 (83.3) & 449 (41.2) & \\
\hline Non-Saudi & $168(16.7)$ & $640(58.8)$ & \\
\hline European & $5(2.9)$ & $3(0.5)$ & \\
\hline American & $2(1.2)$ & $1(0.1)$ & \\
\hline African & $49(29.1)$ & $228(35.6)$ & \\
\hline South East Asian & $69(41.2)$ & $213(33.3)$ & \\
\hline Indian Subcontinent & $43(25.6)$ & $195(30.5)$ & \\
\hline Gender & & & 0.03 \\
\hline Male & $590(58.8)$ & $691(63.4)$ & \\
\hline Female & $413(41.2)$ & $398(36.5)$ & \\
\hline AFB Smear & & & $<0.0001$ \\
\hline Positive & 367 (36.6) & $879(80.7)$ & \\
\hline Negative & $636(63.4)$ & $210(19.3)$ & \\
\hline HIV & & & 0.62 \\
\hline Positive & $4(0.39)$ & $3(0.27)$ & \\
\hline Negative & $632(63.0)$ & $589(54.1)$ & \\
\hline Not Available & $371(37.0)$ & $500(45.9)$ & \\
\hline
\end{tabular}

genetic lineages and 2 sub lineages of lineage 4 (based on large sequence polymorphisms). Interestingly, $M$. africanum lineages West African I (Lineage 5) and West African II (Lineage 6) were also observed. Delhi/CAS (27.4\%) (Lineage 3) was predominant followed by EAI (15.7\%) (Lineage 1), Haarlem (7.5\%) and Ghana (6.8\%). In addition, 119 (11.8\%) isolates of $M$. bovis and 16 (1.6\%) isolates of "undefined" strains have been observed (Fig. 2, Table 2).

\section{Phylogenetic diversity of MTBC isolates in pulmonary tuberculosis}

Pulmonary isolates were also phylogenetically diverse with the presence of six defined genetic lineages of MTBC. West African II lineage was absent among the pulmonary cases. The major identified lineage was Delhi/CAS (26.3\%) followed by Ghana (12.8\%), EAI (12.7\%), and Haarlem (11.7\%) respectively. Interestingly, $89(8.2 \%)$ isolates could not be defined into any strain lineage based on the combined analysis of spoligo and MIRU typing profiles (Fig. 2, Table 2).

\section{Comparison of pulmonary and extrapulmonary TB lineage diversity}

Comparative analysis of pulmonary and EPTB genotypic data showed similar representation of lineages. However, some of the lineages were over represented among certain sites of infections. Lineages- Ghana, Beijing, Haarlem and $\mathrm{S}$ showed a higher affinity towards the pulmonary site of infection. On the other hand, Uganda-I, EAI and Cameroon showed more cases of EPTB. The major lineage among the studied groups Delhi/CAS showed equal rate of presentation in both pulmonary and EPTB cases. M.bovis (78.8\%) was mostly found among EPTB isolates. Although, total number of cases were less, $M$. africanum strains, West African I and II were found more among extrapulmonary cases. 'Ancestral' strains were more common among EPTB (402, 40.1\%) compared to PTB (321, 29.5\%). Furthermore, 'Modern' strains were comparatively higher among PTB cases (679, $62.3 \%$ ) than EPTB (585, 58.3\%) (Fig. 2, Table 2).

\section{Lineages and associating EPTB sites}

We analyzed in detail the adaptability of each major lineage towards different sites of infection. Five major sites such as lymph nodes, gastrointestinal, genitourinary, central nervous systems and bone and joints were analyzed against nine major lineages. Of the 8 major M.tuberculosis lineages analyzed against sites of infection Delhi/CAS, EAI, Beijing, Haarlem, LAM and Ghana showed the capability to cause infection to all the sites. On the other hand, Uganda-I and Cameroon were not observed among the rare sites of infection. M.bovis 


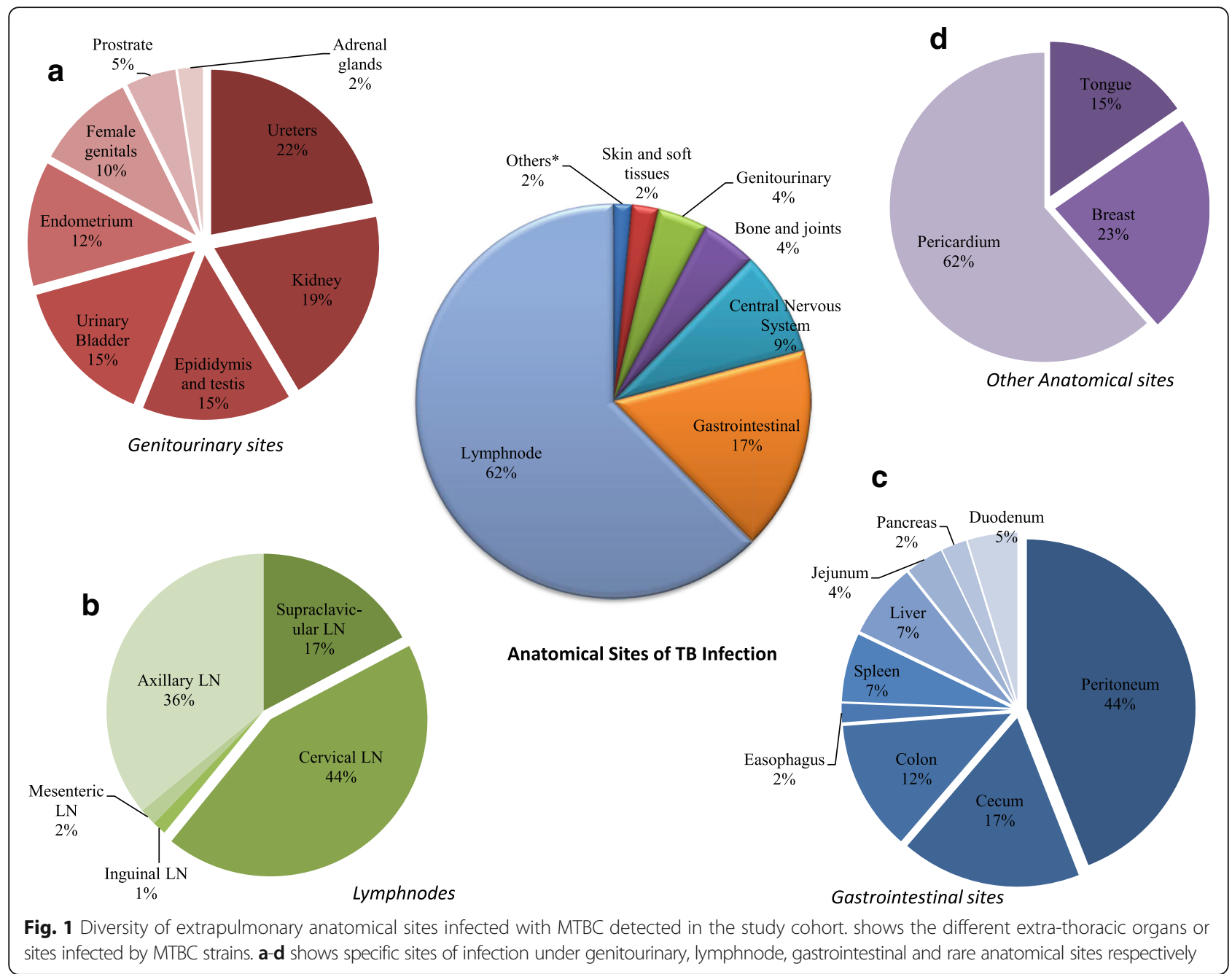

infection was highly confined to lymph nodes (88.2\%), although other sites also involved (Additional file-).

Statistical analysis based on logistic regression was conducted among selected lineages and extrapulmonary sites of infections. A clustered bar-graph was used to depict the variability in the prevalence of sites within each lineage. The chi-square test was used to detect the significance of the difference in such variations (Fig. 3). The results showed the preponderance of certain lineages to major infection sites such as lymph nodes, gastrointestinal and central nervous systems. Delhi/CAS (Indo-oceanic) was noticed with statistical significance to cause infection in lymph nodes $(P$-value $<0.001$; OR $0.57,95 \% \mathrm{CI} 0.411-0.734)$ and organs in gastrointestinal systems ( $P$-value 0.001; OR 1.87, 95\%CI 1.22-2.53). Lineage EAI (East African Indian) also showed a significant association with central nervous system infection sites ( $P$-value 0.04 ; OR $1.98,95 \%$ CI $0.76-3.19)$. East Asian and Euro American lineages showed no association with any of the analyzed infection sites. However, further analysis on Euro American lineages showed,
Uganda-I with an association to gastrointestinal sites ( $P$-value 0.02 ; OR $2.41,95 \%$ CI $0.77-4.06)$. M.bovis also showed statistical significance with, lymph nodes $(P$-value $<0.001$; OR 5.22, 95\% CI 2.23-8.22) and gastrointestinal sites ( $P$-value 0.001; OR 0.33, 95\% CI 0.085-0.567). $M$. africanum lineages West African-I and II were few in numbers, thus a detailed analysis was restricted (Fig. 3; Additional file 3).

\section{Discussion}

Relationships between MTBC phylogenetic lineages and clinical site of TB have been analyzed systematically in a cohort of Saudi Arabian patients for the first time. Ahead of the previous studies which reported a primary data on the site of infection and mycobacterial lineages in the country, the current study congregates with a detailed outcome from a large population of diverse patient cohort of 2092 cases [8]. However, previous international studies also showed inconsistent findings on the association of certain phylogenetic lineages with particular infection sites and disease presentation of tuberculosis $[6,7,12,13]$. 


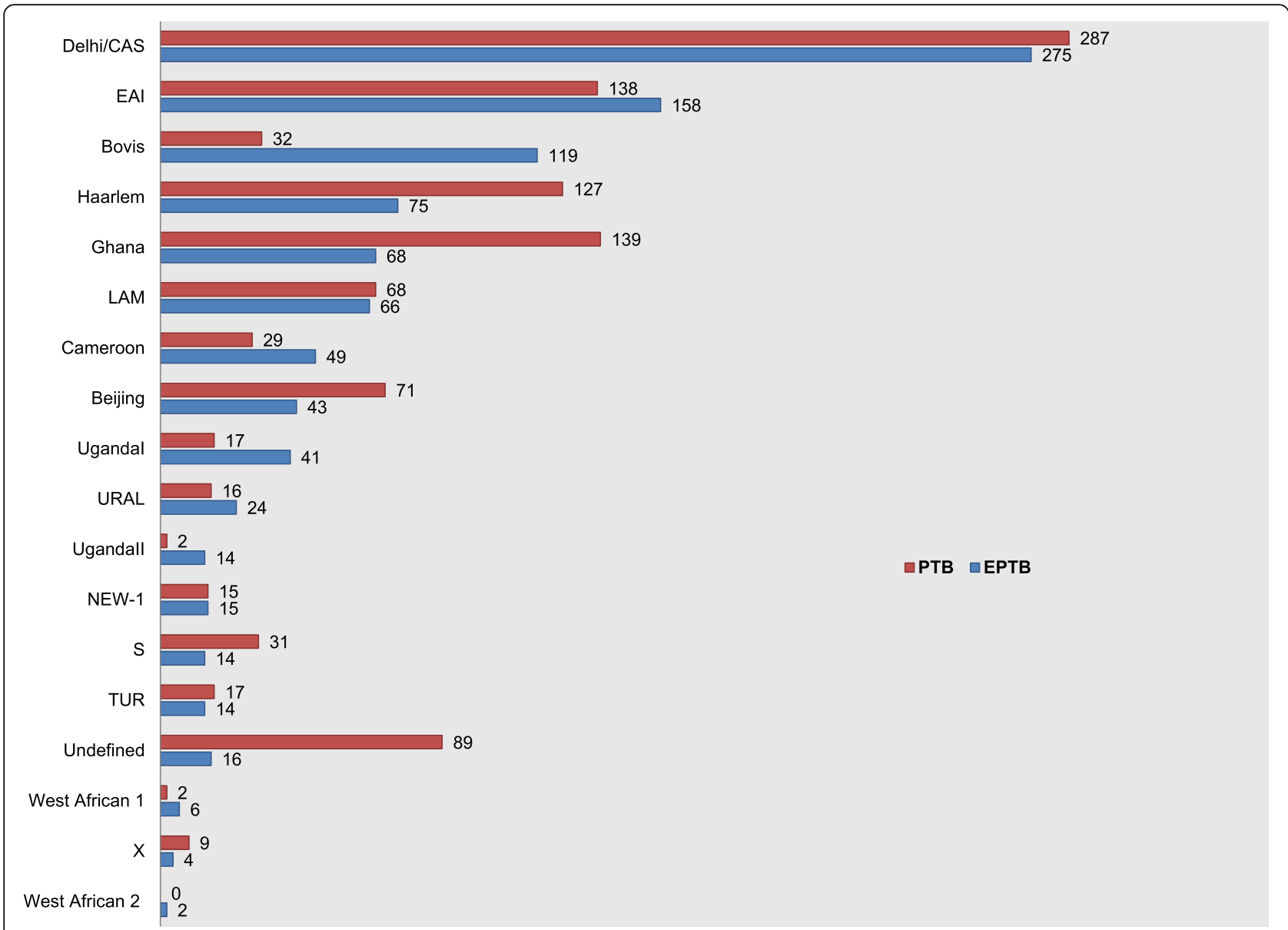

Fig. 2 Comparative Lineage variations identified among extra-pulmonary and pulmonary M.tuberculosis isolates. The figure shows difference in distribution of lineages defined by combined spoligo and 24 loci MIRU VNTR profiles among pulmonary and extra pulmonary anatomical sites

The current findings showed positive association of MTBC lineages (Delhi/CAS, EAI, Uganda-I and M.bovis) with site of infections such as lymph nodes, gastrointestinal and central nervous systems respectively.

Demographical findings of the cohort showed a domination of Saudi nationals. This finding is inversely proportional to the national tuberculosis data where Non-Saudis were reported with more TB incidences [2]. However, the finding is non-conclusive and the expected reason for this variation is that, opted study centers were four referral hospitals including a military facility which provide services largely to citizens only. The gender of study subjects showed predominance of male, which corroborates with the findings of many recent studies which showed similar trend $[2,8,14]$. In addition, localization of TB manifestations strongly depends on several clinical features. Previously younger age is one of the most common associated with extrapulmonary infections [15-17]. The current findings also corroborate with previous studies and showed statistically significant predominance of younger age towards EPTB.
HIV positivity in the cohort was very much limited (0.33\%), although $58.5 \%$ of the total cases only underwent the testing. This finding is in concordance with the low rate of annually reported HIV positive cases among the local population (1.5 cases per 100,000 for Saudis) in the country [18]. HIV testing data was inconsistently recorded (46\% not tested) in the study, while untreated HIV infection is well recognized as an important factor in determining an extrapulmonary clinical phenotype, therefore its influence on clinical phenotype could not be analyzed [17].

The site of infections followed very detailed classification and reported as highly diverse with several rare sites. Lymph node was the most commonly infected sites followed by the gastrointestinal, osteoarticular and central nervous systems. This finding shows similar trend reported in a recent Saudi Arabian and international studies $[3,14,19]$. The current cohort of EPTB cases revealed several rare infection sites such as bone marrow, breast, tongue, testis, kidney, prostate glands, spleen, pancreas, duodenum, cecum and jejunum as observed in available literature $[20,21]$. 
Table 2 Association of pulmonary and extra pulmonary sites against major strain lineages

\begin{tabular}{|c|c|c|c|c|}
\hline \multirow[t]{2}{*}{ Lineages } & \multirow{2}{*}{$\begin{array}{l}\text { Total (N/\%) } \\
N=2092\end{array}$} & \multirow{2}{*}{$\begin{array}{l}\text { N (\%) } \\
\text { PTB }^{1}(N=1089)\end{array}$} & \multirow{2}{*}{$\begin{array}{l}N(\%) \\
\text { EPTB }^{2}(N=1003)\end{array}$} & \multirow[t]{2}{*}{ OR $(95 \% \mathrm{Cl}) / P$ value } \\
\hline & & & & \\
\hline M. bovis & $151(7.3)$ & $32(2.9)$ & $119(11.8)$ & $0.22(0.15-0.33) /<0.0001$ \\
\hline Delhi/CAS & $562(26.9)$ & $287(26.3)$ & $275(27.4)$ & $0.94(0.78-1.15) / 0.58$ \\
\hline$E A l$ & $296(14.2)$ & $138(12.7)$ & $158(15.7)$ & $0.77(0.60-0.99) / 0.04$ \\
\hline Beijing & $114(5.4)$ & $71(6.5)$ & $43(4.3)$ & $1.55(1.05-2.29) / 0.02$ \\
\hline Haarlem & $202(9.6)$ & $127(11.7)$ & $75(7.5)$ & $1.63(1.21-2.20) / 0.001$ \\
\hline LAM & $134(6.4)$ & $68(6.2)$ & $66(6.6)$ & $0.94(0.66-1.34) / 0.75$ \\
\hline Cameroon & $78(3.7)$ & $29(2.7)$ & $49(4.9)$ & $0.53(0.33-0.85) / 0.008$ \\
\hline Ghana & $207(9.9)$ & $139(12.8)$ & $68(6.8)$ & $2.01(1.48-2.71) /<0.0001$ \\
\hline Uganda I & $58(2.8)$ & $17(1.6)$ & $41(4.1)$ & $0.37(0.21-0.66) / 0.0007$ \\
\hline URAL & $40(1.9)$ & $16(1.5)$ & $24(2.4)$ & $0.60(0.32-1.1) / 0.127$ \\
\hline TUR & $31(1.5)$ & $17(1.6)$ & $14(1.4)$ & $1.12(0.55-2.28) / 0.75$ \\
\hline S & $45(2.1)$ & $31(2.8)$ & $14(1.4)$ & $2.07(1.09-3.91) / 0.025$ \\
\hline Uganda II & $16(0.76)$ & $2(0.2)$ & $14(1.4)$ & $0.13(0.03-0.57) / 0.007$ \\
\hline New-l & $30(1.4)$ & $15(1.4)$ & $15(1.5)$ & $0.92(0.44-1.89) / 0.82$ \\
\hline Others $^{3}$ & $144(6.9)$ & $100(9.2)$ & $28(2.8)$ & - \\
\hline
\end{tabular}

${ }^{1}$ Pulmonary tuberculosis

${ }^{2}$ Extrapulmonary tuberculosis

${ }^{3}$ Includes lineages, West African I and II, X, New-I and undefined lineages

The major advantage of our data compared to previously published studies with limited sample size and sample diversity was the presence of all defined lineages. Phylogenetic diversity of pulmonary and extrapulmonary isolates showed the presence of all defined MTBC lineages (Lineage 1-7) in the country. Interestingly, for the first time in the country, presence of $M$. africanum lineages West African I and II were noticed. M.africanum has not been detected in any of the previous nationwide studies [8-10]. Saudi Arabia annually receives approximately 8-10 millions of pilgrims (from 184 countries) from $\mathrm{TB}$ endemic regions, in addition to its migrant workers population of 10.4 million from around the world. This massive influx of foreign nationals solely induces a higher impact on the population structure of MTBC in the country [10, 22]. The increased possibilities of TB transmission and exportation in mass gathering particularly during Hajj were highly projected [23]. Therefore, the higher diversity of strain lineages in the study could be well explained.

Comparative analysis on the strain diversity between PTB and EPTB was a key objective of the study. The findings showed the presence of all the lineages among both groups though the proportion varied. Ancestral lineages were found relatively high among EPTB cases, which are in concordance with previously published studies from other part of the world including TB endemic regions [24, 25]. On the other hand, "Modern" strains were more common among PTB. Predominance of "Modern" strains among the pulmonary cases in the country was previously well documented, corroborating with other global regions $[6,8,13]$.

Detailed statistical analysis to find an organ or site specific preponderance of MTBC lineages showed significant association of ancestral lineages, Delhi/CAS and M.bovis to lymphadenopathy and gastrointestinal TB. Although, previous studies showed predominant isolation of Delhi/CAS and M.bovis from lymph nodes and gastrointestinal sites only few of them were statistically significant [24, 26, 27]. Interestingly, association of lineage EAI with central nervous system TB which mainly included tuberculous meningitis (TBM) was significant. Although previous studies showed the mycobacterial genotypes could play a major role in disease severity, emergence of drug resistance, host response and transmissibility in TBM cases, findings of statistical significance between certain lineages and CNS disease sites are highly scarce $[28,29]$. Association of Uganda-I lineage to gastro intestinal sites of infection was another significant finding. The Uganda-I lineage has not been studied in detail as a cause of EPTB and therefore very much limited information is only available in literature on its association with any clinical manifestation [30].

The influence of genomic diversity on pathobiological properties such as transmissibility, virulence, immune responses, and clinical manifestations were established in several previous studies $[6,31,32]$. In addition, lineage specific difference in mycobacterial transcriptomic responses, cytokine induction patterns in animal or cellular infection models also were proved in recent 


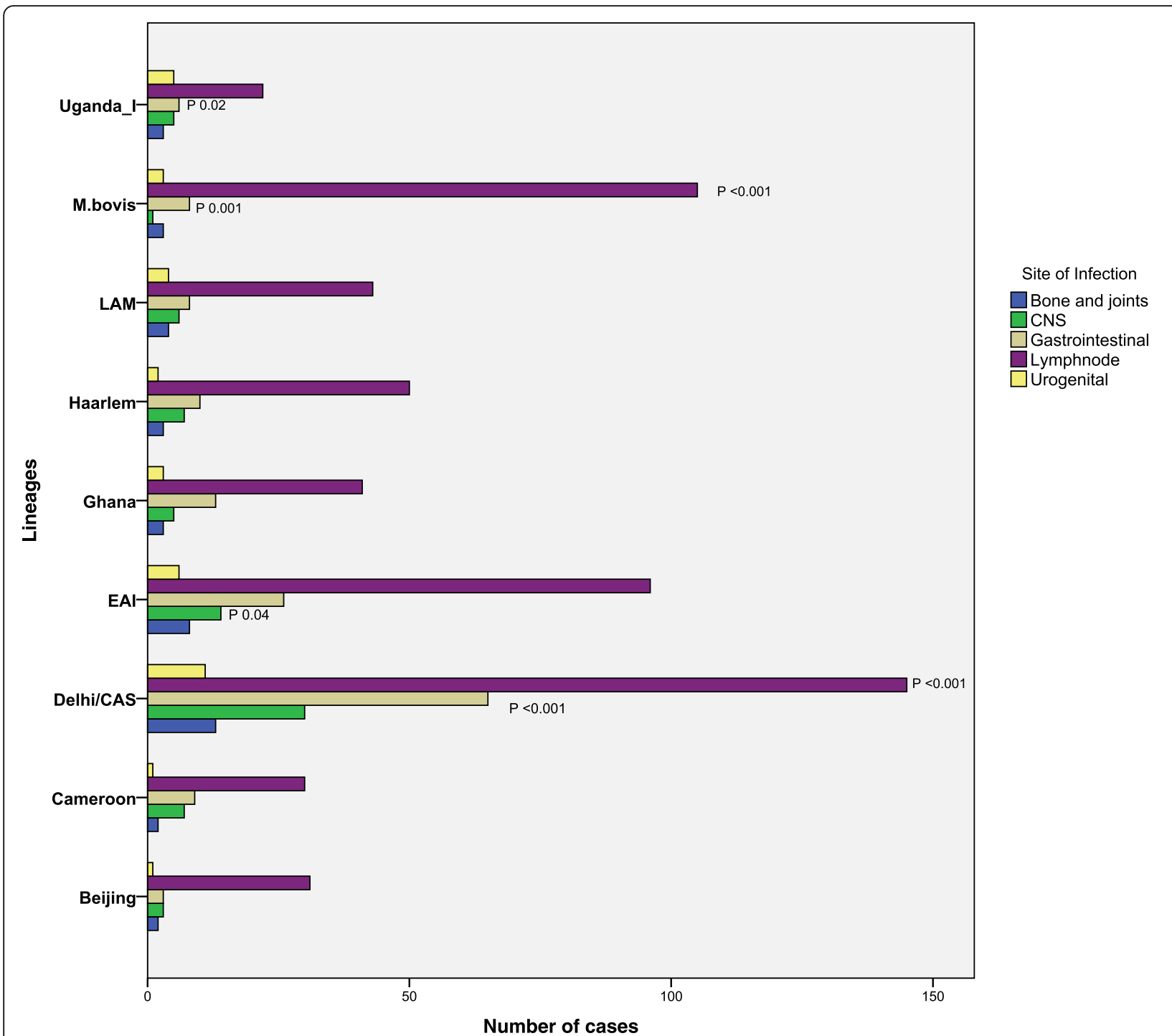

Fig. 3 Statistical variability in the prevalence of sites within each lineage. The clustered bar-graph was prepared after chi-square testing with Bonferroni correction for multiplicity to detect the significance of differences in such variations

studies [33-35]. One of the most intensively studied lineage Beijing, showed several potential mechanisms as evidences of associations towards clinical manifestations [36]. In animal models less protective Th1 response and high virulence has been documented when infected with Beijing strains [35]. Other proposed mechanisms in Beijing strains, which increase the pathobiological adaptations and phenotypic stability are DosR up- regulation and production of a phenolic glycolipid by an intact pks15-1 [37, 38]. However, the real mechanisms behind all such associations particularly on less frequently encountered lineages are still not well established.

The study has few limitations mostly related to sampling and data analysis. Although, the study population largely consisted of Saudi nationals it cannot be considered as a nationwide population based analysis. The cohort was a selective group of citizens and mostly from the central region of the country. The expatriate population was not well represented mainly due to their eligibility of treatment in study centers although they represent $33 \%$ of total population of the country. Analysis of confounding factors behind the EPTB in the current cohorts was limited and this was not included as a major objective of the study. In addition, $5.1 \%$ of the total isolates in the study could not be assigned into "defined" lineage even after combining the MIRU and spoligotyping techniques, and no alternative technique was utilized to define their phylogenetic nature.

\section{Conclusions}

In conclusion, as the first systematic large study of its type from the Middle East, the findings showed the 
extreme genotypic diversity of MTBC in extrapulmonary sites of infections. Statistically significant association of infection sites with lineages such as Delhi/CAS, EAI, Uganda-I and M.bovis could be established. Moreover, these findings support the view that MTBC strains within individual genotypic lineages might have evolved unique pathogenic characteristics that are capable of influencing the clinical outcome of the infection.

\section{Additional files}

Additional file 1: Nationality stratified by site of infection. Table shows the distribution of pulmonary and extrapulmonary cases among 39 nationalities included in the study. (PDF $194 \mathrm{~kb}$ )

Additional file 2: Nationality of the patient and distribution of lineages. The figure shows the distribution of major MTBC lineages among top 11 nationalities. (PDF $277 \mathrm{~kb}$ )

Additional file 3: Statistical association of major MTBC lineages and extrapulmonary site of infections. (PDF $112 \mathrm{~kb}$ )

\section{Abbreviations}

CAS: Central Asian; EAl: East African Indian; EPTB: Extrapulmonary tuberculosis; MIRU: Mycobacterial Interspersed Repetitive Units; MTBC: Mycobacterium tuberculosis complex; PTB: Pulmonary tuberculosis; TB: Tuberculosis; TBM: Tuberculous meningitis

\section{Acknowledgements}

We acknowledge the timely help of Dr. Abdulhameed Hadi Al Faqih (KFMC, Riyadh) and Dr.Abdulrahman Abdullah Korairi (KFMC, Riyadh) in clinical data collection.

\section{Funding}

The study has been funded by King Abdulaziz City for Science and Technology, Riyadh, Saudi Arabia under Grant Code \# AT-34-103.

\section{Availability of data and materials}

The datasets generated and/or analyzed during the current study are available from the corresponding author on reasonable request.

\section{Authors' contributions}

Conception and design of the study: BV, SA, AA. Sample and data collection, reviewing manuscript: $A B, M E, S A J, S A T, N E, H A$; Data management and interpretation: BV, MS. Drafting the manuscript: BV. All the authors reviewed and approved final version of the manuscript.

\section{Ethics approval and consent to participate}

The study has been reviewed and approved by Office of Research Affairs (ORA) at King Faisal Specialist Hospital and Research Centre (KFSHRC), Riyadh. Since this study is a retrospective clinical study the need for written consent was formally waved by ORA at KFSHRC, Riyadh, Saudi Arabia.

\section{Consent for publication}

Not applicable

\section{Competing interests}

The authors declare that they have no competing interests.

\section{Publisher's Note}

Springer Nature remains neutral with regard to jurisdictional claims in published maps and institutional affiliations.

\section{Author details}

${ }^{1}$ Department of Infection and Immunity, MBC-03, King Faisal Specialist Hospital and Research Centre, Post Box \# 3354, Riyadh 11211, Saudi Arabia. ${ }^{2}$ Medical Specialties Department, King Fahad Medical City, Riyadh, Saudi Arabia. ${ }^{3}$ Department of Medicine, King Faisal Specialist Hospital and Research
Centre, Riyadh, Saudi Arabia. ${ }^{4}$ Department of Microbiology, King Abdul Aziz Medical City, Riyadh, Saudi Arabia. ${ }^{5}$ Department of Medicine, Prince Sultan Military Medical City, Riyadh, Saudi Arabia. ${ }^{6}$ Department of Pathology and Laboratory Medicine, King Faisal Specialist Hospital and Research Centre, Riyadh, Saudi Arabia. ${ }^{7}$ Department of Microbiology, Prince Sultan Military Medical City, Riyadh, Saudi Arabia. ${ }^{8}$ National Biotechnology Centre, King Faisal Specialist Hospital and Research Centre, Riyadh, Saudi Arabia.

Received: 1 July 2018 Accepted: 16 December 2018

Published online: 27 December 2018

\section{References}

1. World Health Organization, Global Tuberculosis Report, 2017. WHO, Geneva, Switzerland, 2017.

2. Ministry of Health Saudi Arabia: Statistical year Book-1437. MOH, Riyadh, KSA- 2016

3. Kruijshaar ME, Abubakar I. Increase in extrapulmonary tuberculosis in England and Wales 1999-2006. Thorax. 2009;64:1090.

4. Sandgren A, Hollo V, van der Werf MJ. Extrapulmonary tuberculosis in the European Union and European economic area, 2002 to 2011. Euro Surveill. 2013;18(12).

5. Houston A, Macallan DC. Extrapulmonary tuberculosis. Medicine. 2014;42: $18-22$.

6. Caws M, Thwaites G, Dunstan S, Hawn TR, Lan NT, Thuong NT, et al. The influence of host and bacterial genotype on the development of disseminated disease with Mycobacterium tuberculosis. PLoS Pathog. 2008;4:e1000034.

7. Firdessa R, Berg S, Hailu E, Schelling E, Gumi B, Erenso G, et al. Mycobacterial lineages causing pulmonary and extrapulmonary tuberculosis, Ethiopia. Emerg Infect Dis. 2013;19:460-3.

8. Al-Hajoj S, Varghese B, Al-Habobe F, Shoukri MM, Mulder A, van Soolingen D. Current trends of Mycobacterium tuberculosis molecular epidemiology in Saudi Arabia and associated demographical factors. Infect Genet Evol. 2013; 16:362-8.

9. Varghese B, Supply P, Allix-Beguec C, Shoukri M, Al-Omari R, Herbawi M, et al. Admixed phylogenetic distribution of drug resistant Mycobacterium tuberculosis in Saudi Arabia. PLoS One. 2013;8:e55598.

10. Varghese B, Supply P, Shoukri M, Allix-Beguec C, Memish Z, Abuljadayel N, et al. Tuberculosis transmission among immigrants and autochthonous populations of the eastern province of Saudi Arabia. PLoS One. 2013;8: e77635.

11. Gagneux S, Small PM. Global phylogeography of Mycobacterium tuberculosis and implications for tuberculosis product development. Lancet Infect Dis. 2007;7:328-37.

12. Nicol MP, Wilkinson RJ. The clinical consequences of strain diversity in Mycobacterium tuberculosis. Trans R Soc Trop Med Hyg. 2008;102:955-65.

13. Pareek M, Evans J, Innes J, Smith G, Hingley-Wilson S, Lougheed KE, et al. Ethnicity and mycobacterial lineage as determinants of tuberculosis disease phenotype. Thorax. 2013;68:221-9.

14. Al-Hajoj S, Shoukri M, Memish Z, AlHakeem R, AlRabiah F, Varghese B. Exploring the sociodemographic and clinical features of extrapulmonary tuberculosis in Saudi Arabia. PLoS One. 2015;10:e0101667.

15. Sreeramareddy CT, Panduru KV, Verma SC, Joshi HS, Bates MN. Comparison of pulmonary and extrapulmonary tuberculosis in Nepal- a hospital-based retrospective study. BMC Infect Dis. 2008;8:8

16. Memish ZA, Bamgboye EA, Abuljadayel N, Smadi H, Abouzeid MS, Al Hakeem RF. Incidence of and risk factors associated with pulmonary and extra-pulmonary tuberculosis in Saudi Arabia (2010-2011). PLoS One. 2014;9:e95654

17. Yang Z, Kong Y, Wilson F, Foxman B, Fowler AH, Marrs CF, et al. Identification of risk factors for extrapulmonary tuberculosis. Clin Infect Dis. 2004;38:199-205.

18. Mazroa MA, Kabbash IA, Felemban SM, Stephens GM, Al-Hakeem RF, Zumla Al, et al. HIV case notification rates in the Kingdom of Saudi Arabia over the past decade (2000-2009). PLoS One. 2012;7:e45919.

19. Solovic I, Jonsson J, Korzeniewska-Kosela M, Chiotan DI, Pace-Asciak A, Slump E, et al. Challenges in diagnosing extrapulmonary tuberculosis in the European Union, 2011. Euro Surveill. 2013;18(12).

20. Debi U, Ravisankar V, Prasad KK, Sinha SK, Sharma AK. Abdominal tuberculosis of the gastrointestinal tract: revisited. World J Gastroenterol. 2014; 20:14831-40.

21. Jain P, Jain I. Oral manifestations of tuberculosis: step towards early diagnosis. J Clin Diagnost Res. 2014;8:ZE18-21. 
22. Al-Hajoj SA, Zozio T, Al-Rabiah F, Mohammad V, Al-Nasser M, Sola C, et al. First insight into the population structure of Mycobacterium tuberculosis in Saudi Arabia. J Clin Microbiol. 2007:45:2467-73.

23. Zumla A, Saeed AB, Alotaibi B, Yezli S, Dar O, Bieh K, et al. Tuberculosis and mass gatherings-opportunities for defining burden, transmission risk, and the optimal surveillance, prevention, and control measures at the annual hajj pilgrimage. Int J Infect Dis. 2016;47:86-91.

24. Sankar MM, Singh J, Diana SC, Singh S. Molecular characterization of Mycobacterium tuberculosis isolates from north Indian patients with extrapulmonary tuberculosis. Tuberculosis (Edinb). 2013;93:75-83.

25. Svensson E, Millet J, Lindqvist A, Olsson M, Ridell M, Rastogi N. Impact of immigration on tuberculosis epidemiology in a low-incidence country. Clin Microbiol Infect. 2011;17:881-7.

26. Biadglegne F, Merker M, Sack U, Rodloff AC, Niemann S. Tuberculous lymphadenitis in Ethiopia predominantly caused by strains belonging to the Delhi/CAS lineage and newly identified Ethiopian clades of the Mycobacterium tuberculosis complex. PLoS One. 2015;10:e0137865.

27. Lari N, Rindi L, Cristofani R, Rastogi N, Tortoli E, Garzelli C. Association of Mycobacterium tuberculosis complex isolates of BOVIS and central Asian (CAS) genotypic lineages with extrapulmonary disease. Clin Microbiol Infect. 2009;15:538-43.

28. Ford CB, Shah RR, Maeda MK, Gagneux S, Murray MB, Cohen T, et al. Mycobacterium tuberculosis mutation rate estimates from different lineages predict substantial differences in the emergence of drug-resistant tuberculosis. Nat Genet. 2013:45:784-90.

29. Thwaites G, Caws M, Chau TT, D'Sa A, Lan NT, Huyen MN, et al. Relationship between Mycobacterium tuberculosis genotype and the clinical phenotype of pulmonary and meningeal tuberculosis. J Clin Microbiol. 2008:46:1363-8.

30. Wamala D, Asiimwe B, Kigozi E, Mboowa G, Joloba M, Kallenius G. Clinicopathological features of tuberculosis due to Mycobacterium tuberculosis Uganda genotype in patients with tuberculous lymphadenitis: a cross sectional study. BMC Clin Pathol. 2014;14:14.

31. de Jong BC, Hill PC, Brookes RH, Gagneux S, Jeffries DJ, Otu JK, et al. Mycobacterium africanum elicits an attenuated $T$ cell response to early secreted antigenic target, $6 \mathrm{kDa}$, in patients with tuberculosis and their household contacts. J Infect Dis. 2006;193:1279-86.

32. Rakotosamimanana N, Raharimanga V, Andriamandimby SF, Soares JL, Doherty TM, Ratsitorahina M, et al. Variation in gamma interferon responses to different infecting strains of Mycobacterium tuberculosis in acid-fast bacillus smear-positive patients and household contacts in Antananarivo, Madagascar. Clin Vaccine Immunol. 2010;17:1094-103.

33. Portevin D, Gagneux S, Comas I, Young D. Human macrophage responses to clinical isolates from the Mycobacterium tuberculosis complex discriminate between ancient and modern lineages. PLoS Pathog. 2011;7:e1001307.

34. Homolka S, Niemann S, Russell DG, Rohde KH. Functional genetic diversity among Mycobacterium tuberculosis complex clinical isolates: delineation of conserved core and lineage-specific transcriptomes during intracellular survival. PLoS Pathog. 2010;6:e1000988.

35. Lopez B, Aguilar D, Orozco H, Burger M, Espitia C, Ritacco V, et al. A marked difference in pathogenesis and immune response induced by different Mycobacterium tuberculosis genotypes. Clin Exp Immunol. 2003;133:30-7.

36. Parwati I, van Crevel R, van Soolingen D. Possible underlying mechanisms for successful emergence of the Mycobacterium tuberculosis Beijing genotype strains. Lancet Infect Dis. 2010;10:103-11.

37. Reed MB, Gagneux S, Deriemer K, Small PM, Barry CE 3rd. The W-Beijing lineage of Mycobacterium tuberculosis overproduces triglycerides and has the DosR dormancy regulon constitutively upregulated. J Bacteriol. 2007; 189:2583-9.

38. Sinsimer D, Huet G, Manca C, Tsenova L, Koo M-S, Kurepina N et al. The phenolic glycolipid of Mycobacterium tuberculosis differentially modulates the early host cytokine response but does not in itself confer hypervirulence. Infect Immunity. 2008;76:3027-36.

Ready to submit your research? Choose BMC and benefit from:

- fast, convenient online submission

- thorough peer review by experienced researchers in your field

- rapid publication on acceptance

- support for research data, including large and complex data types

- gold Open Access which fosters wider collaboration and increased citations

- maximum visibility for your research: over $100 \mathrm{M}$ website views per year

At BMC, research is always in progress.

Learn more biomedcentral.com/submissions 